\title{
Analysis and Simulation of a Mathematical Model of Ebola Virus Dynamics in vivo
}

\author{
Thomas Wester* \\ Faculty Advisers: Sonia Garcia*, Daniel Isaac ${ }^{+}$ \\ * Department of Mathematics, U.S. Naval Academy, 572C Holloway Rd Annapolis MD 21402 (m166912@usna.edu, smg@usna.edu) \\ + Department of Chemistry, U.S. Naval Academy, 572M Holloway Rd Annapolis MD 21402, (isaac@usna.edu)
}

July 31, 2015

\begin{abstract}
Ebola is known to evade detection by the immune system during infection. In this paper, we use mathematical modeling as a tool to investigate and analyze the immune system dynamics in the presence of Ebola virus infection. The resulting model is a system of non-linear ordinary differential equations derived from known biological dynamics and a few biologically reasonable assumptions. In this paper, we prove existence and uniqueness as well as positivity and boundedness of the solutions to the differential equations. In addition, we derive the viral and immune reproduction numbers, and analyze the local asymptotic stability of the differential equation model. Furthermore, we run numerical simulations to illustrate the impact the variation of the parameters has on the behavior of the system. The analysis we develop provides thresholds for both determining the persistence and elimination of Ebola virus from the immune system, and represents the known biological dynamics of Ebola virus infection.
\end{abstract}

Keywords: Mathematical Modeling, Ebola Virus, Stability, Numerical Simulations

\section{Introduction}

A mathematical model is a platform for understanding the behavior of a dynamical system. The goal of this paper is to use mathematical modeling as a tool to examine and analyze the viral dynamics of the Ebola virus in vivo. We begin by providing a background of the necessary biology in order to understand the model and results. In Section 2, we utilize the current understanding of the dynamics of Ebola virus infection and apply biologically reasonable assumptions to motivate a system of non-linear ordinary differential equations and describe the interactions between pertinent cellular and viral populations. In Section 3, we outline existence, uniqueness, positivity, and boundedness of solutions to the initial value problem. Then, in Section 4, we analyze the system of differential equations to gain insights into the local asymptotic stability of the system and allow for predictions of behavior based on parameter variation. Furthermore, we conduct numerical simulations to illustrate the behavior of the system under differing parameter values. Section 5 provides discussion of results and conclusions based on our analysis. Our goal is to develop a better understanding of the behavior of the Ebola virus to extract experimental results which are extremely difficult to obtain using a traditional biological approach. In addition, we intend to contextualize viral infection in specific populations in order to further our understanding of Ebola virus dynamics and their relationship to the effectiveness (or lack thereof) of the immune response the virus elicits.

\subsection{Biological Background}

\subsubsection{Ebola Virus}

Ebola virus is one of the deadliest human pathogens currently known and is the cause of the 2013-2015 Ebola epidemic in West Africa which has resulted in at least 23,014 total cases and 9,840 total deaths as of March 4, 2015 according to the Center for Disease Control [5]. Ebola virus produces one of the most lethal 


\section{INTRODUCTION}

forms of hemorrhagic fever. As a result, the virus maintains mortality rates between $40 \%$ and $90 \%$, averaging about $50 \%$ and can cause death in less than two days [18]. The rapid progression of Ebola virus infection has further complicated the control of the disease, offering little opportunity to develop acquired immunity [18]. In addition, deficiencies in specific and non-specific immune responses result in unrestricted viral replication and dissemination in the host, causing death typically within 10 days after the appearance of symptoms [1,3].

Ebola virus is a member of the filoviridae family of viruses. This family of viruses is comprised of singlestranded negative-sense RNA viruses that also includes Marburg virus, a virus responsible for two deadly outbreaks in the last 15 years. Of the five identified strains of Ebola virus, four - Zaire ebolavirus, Tai Forest ebolavirus (Ivory Coast), Bundibugyo ebolavirus, and Sudan ebolavirus strains have been shown to cause infection in humans [18]. Ebola virus can be contracted through abrasions and lesions in the skin after handling fluids or tissues from an infected patient $[1,3]$. However, there is also evidence that mucosal exposure via the airborne route is possible in some susceptible animal species.

The Ebola virus cellular tropism consists largely of immune cells (monocytes, macrophages and dendritic cells) which all serve as initial sites for viral infection and replication [1]. Each Ebola virus virion consists of a nucleocapsid containing a negative ssRNA genome surrounded by the nucleoprotein, the polymerase cofactor VP35, the virus specific transcription activator VP30, and the viral RNA polymerase L proteins [3]. In addition, the matrix between the outer viral envelope and the nucleocapsid contains the VP40 and VP24 viral proteins [3].

Ebola virus initiates infection via interactions with cell-surface receptors which are typically $\mathrm{Ca}^{+2}$-dependent ("C-type") lectins. Upon fusion of the viral envelope and the host cell plasma membrane, Ebola virus virions are trafficked to acidic lysosomal and endosomal compartments where cleavage of the viral envelope glycoprotein (GP) is carried out. This cleavage facilitates viral fusion to intracellular membranes and causes the subsequent release of the viral nucleocapsid containing the viral ssRNA. The virally-encoded VP35 polymerase partially un-coats the capsid allowing the polymerase to transcribe the negative-strand ssRNA into positive-stranded mRNAs suitable for translation into the viral proteins required for self-assembly. This same polymerase can then replicate the viral genome many times. Viral genomes and proteins subsequently self-assemble in the infected cell producing substantial numbers of Ebola virus. The virally encoded VP40 protein promotes both the localization of Ebola virus to and budding from the plasma membrane releasing viral particles capable of continuing the infectious cycle.

\subsubsection{Host Immune Response}

Prior to the development of the mathematical model, it is crucial to understand how the immune system responds to infection. This is because survival from Ebola virus infection depends on the host's ability to develop and manifest a robust immune system response early after the introduction of the virus.

A primary component of the immune system is the T-cell which is a lymphocyte that matures in the thymus. T-cell activation is one of the central events in the initiation of an adaptive immune response. T-cells exist in two distinct populations within the immune system, Helper T-cells and Cytotoxic T-lymphocytes (CTLs, also known as Cytolytic T-cells, Cytotoxic T-cells, or Killer T-cells). The two T-cell classes are distinguished by a specific cell-surface protein - CD4 on the helper T-cell and CD8 on the CTL.

The interaction between a naïve T-cell and an antigen presenting cell (APC), such as a dendritic cell or macrophage, initiates the immune response. Upon recognition of a pathogen, APCs process and present peptides from the pathogen on their surface using either Major Histocompatibility Complex (MHC) Class I or Class II molecules. Both Helper T-cells and CTLs contain specialized antibody-like receptors that recognize these molecules; the CD4 protein of the Helper T-cell recognizes and binds to the peptide presented by MHC Class II molecules whereas the CD8 protein expressed by the CTL recognizes and binds to the peptide presented by MHC Class I molecules. Upon these binding events, the CTL become activated.

Activated Helper T-cells serve as the alarm system of the immune system. Their activation causes them to secrete cytokines and proliferate. Cytokines are a chemical mediator that serve as the communication network for the immune system. In addition, cytokines secreted by Helper T-cells play a large role in the activation and proliferation of CTLs. When a CTL is exposed to the cytokines released by the activated Helper T-cell, the CTL itself becomes activated [20]. Upon activation, T-cells exhibiting the CD8 protein are referred to as 
CTLs. Once activated, CTLs maintain the ability to kill the infected cell that the Helper T-cells discriminate as harmful. This occurs when the CTL binds to the target cell and releases a potent chemical called perforin. Perforin perforates the cell membrane of the infected cells and causes the cells to lyse and die [20].

CTL activation is the central event in the initiation of the adaptive immune response [21] and it is crucial to examine the stimulation and effects of CTLs when analyzing the course of infection. Furthermore, the CTL response is a primary component of host survival and recovery during a viral infection. This is due to the CTL's ability to kill compromised cells on contact. In addition, there is evidence that a robust, specific adaptive immune response is required for survival from Ebola virus infections [1]. Comparison of immune responses of survivors during two outbreaks in Gabon in 1996 revealed that survivors and asymptomatic patients have increased number of T-cells and an early CTL mediated response [1]. However, recent characterization of immune responses to Ebola virus infections has revealed that Ebola virus has developed several methods to effectively subvert and counter both the innate and adaptive immune responses $[1,12,3]$. In this paper, we will expand current analysis by considering a CTL response to the introduction of the virus as well as the ability of Ebola virus to evade detection by the host immune system.

\subsection{Motivation}

Ebola virus is a class A bioterrorism and level four biosafety agent [3]. Thus, research using Ebola viruses requires facilities with the utmost levels of containment, strict controls on access, and highly trained personnel. Due to the most recent outbreak of Ebola in Western Africa and the threat it poses to the rest of the world, research into how Ebola interacts with the host, and more specifically the immune system, has significantly increased. This research has led to the development of new insights into how the virus functions and effects immune system behavior [1]. In this paper, we will apply mathematical analysis of a model for Ebola viral dynamics to gain further insights and understanding of viral dynamics during the course of infection.

\section{Ebola Virus Dynamics Model}

In 2010 Banton, Roth, and Pavlovic proposed that the Herz model for viral reproduction could serve as a basis for investigations into the behavior of Ebola virus dynamics in vivo [2]. Furthermore, they suggested that in a basic immune response, infected cells can be assumed to be killed by CTLs [2]. Truckwell modified the Herz system by introducing an additional compartment to account for the CTL response in basic viral infection [24]. In this paper, we implement this modification of the Herz model to explore the immune response to Ebola virus infection via CTL response.

The model considers four distinct populations which are denoted:

$X(t)$ : density of uninfected cells at time $t$,

$I(t)$ : density of infected cells at time $t$,

$V(t)$ : density of virus at time $t$,

$T(t)$ : density of cytotoxic T-lymphocytes at time $t$.

Thus, we consider the mathematical model of Ebola virus infection with an immune response given by the following four-dimensional non-linear system of ordinary differential equations.

(1a) $\frac{d X}{d t}=\lambda-\mu X(t)-\beta V(t) X(t)$
(1b) $\frac{d I}{d t}=\beta V(t) X(t)-\rho I(t) T(t)-\alpha I(t)$
(1c) $\frac{d V}{d t}=c I(t)-\gamma V(t)$
(1d) $\frac{d T}{d t}=\rho I(t) T(t)-\delta T(t)$

With initial conditions $X(0)=X_{0}, I(0)=I_{0}, V(0)=V_{0}, T(0)=T_{0}$. 


\begin{tabular}{|c|c|c|c|c|}
\hline Parameter & Units & Relevant Biological Description & Estimated Value Range & Source \\
\hline$\lambda$ & $\frac{\text { cells }}{m l \times \text { days }}$ & Growth Rate (Uninfected cells) & $0.1-10$ & {$[21,20,24,22]$} \\
\hline$\mu$ & $\frac{1}{d a y s}$ & Death Rate (Uninfected cells) & $0.02-0.03$ & {$[20,7]$} \\
\hline$\beta$ & $\frac{m l}{\text { cells } \times \text { days }}$ & Interaction Rate (Virus and Uninfected cells) & $0.001-0.02$ & {$[20,7,22]$} \\
\hline$\rho$ & $\frac{m l}{\text { cells } \times \text { days }}$ & Interaction Rate (Infected cells and CTLs) & 0.1 & {$[2]$} \\
\hline$\alpha$ & $\frac{1}{d a y s}$ & Death Rate (Infected Cells) & $0.2-0.7$ & {$[15,16,20,7]$} \\
\hline$c$ & $\frac{1}{d a y s}$ & Growth Rate (Virus) & $20-50$ & {$[15,20,24,7]$} \\
\hline$\gamma$ & $\frac{1}{d a y s}$ & Death Rate (Virus) & $2.4-3.8$ & {$[15,22,7,20]$} \\
\hline$\delta$ & $\frac{1}{d a y s}$ & Death Rate (CTLs) & $0.3-0.5$ & {$[16,20,22]$} \\
\hline
\end{tabular}

Table 1: A table denoting the individual parameter, units, biologically relevant description, as well as estimated value range.

A schematic of the biological mechanism of Ebola virus infection in vivo which illustrates the dynamics of the model is shown in Figure 1. In addition, Table 1 provides a biological interpretation of the parameters located within the model as well as estimated values based on previous work. Now we will motivate each equation within the system.

\subsection{Model Development}

Equation 1a models the dynamics of the uninfected cellular population. The equation can be represented by production rate, infection rate, and death rate. The equation is determined to be:

Rate of change of uninfected

cell population $=($ Production rate $)-($ Infection rate $)-($ Death rate $)$

Production rate: We assume that uninfected cells are produced at a constant rate, the uninfected cell growth rate, $\lambda$ $[15,16,21]$.

Infection rate: Uninfected cells can be eliminated by becoming infected by the virus. The interaction between the virus and the uninfected cells is widely known as the mass action principle [16]. This principle describes, from a mathematical perspective, the rate at which the virus infects uninfected cells. The mass action principle results in a term which suggests that the rate of interaction between the virus and uninfected cells, $\beta$, is directly proportional to the product of the participating populations $[15,16]$.

Death rate: The uninfected cell death rate term is determined by the elimination of uninfected cells not due to infection as a result of the virus. The death rate, $\mu$, can be assumed to be proportional to the uninfected cell population [15].

Equation $1 \mathbf{b}$ represents the dynamics of infected cells. The equation for the rate of change of the infected cell population is dictated by both the rate of infection and death rate. The equation can be represented as:

$$
\text { Rate of change of infected cell population }=(\text { Infection rate })-(\text { Interaction rate })-(\text { Death rate })
$$

Infection rate: This term is the same as the infection rate term in the uninfected cell differential equation with a reversal in sign. This is a result of the fact that the only way that infected cells can be created is by infecting previously uninfected cells [16]. The human immune system does not naturally produce infected cells; 


\section{ANALYSIS OF THE MODEL}

thus, the term remains the same as previously determined.

Interaction rate: CTLs are activated when contact between a CTL and an infected cell is made within the infected host. This interaction causes the infected cell to be removed from the infected cell population since it no longer maintains the ability to infect previously uninfected cells. The interaction rate for the activated CTLs, $\rho$, can be assumed to be proportional to the product of the CTL and infected cell populations.

Death rate: Similar to uninfected cell death, infected cells are cleared by the immune system at a rate, $\alpha$, proportional to the uninfected cell population [16].

Equation 1c mathematically describes the dynamics of the virus cell population. This equation consists of the virus production rate and viral clearance rate and is:

$$
\text { Rate of change of virus population }=(\text { Growth rate })-(\text { Clearance rate })
$$

Production rate: While the virus production rate varies from cell to cell and individual to individual, when considering the aggregate population this model assumes the rate of proliferation is constant and that new viruses are produced at a rate, $c$, proportional to the infected cell population [16].

Clearance rate: There are two manners in which the virus-infected cells are eliminated, viral cytopathic effects and immune-mediated cellular destruction [11]. Viral cytopathic effects occur as a result of the virus infecting healthy uninfected cells. When a viral particle infects a previously uninfected cell, the viral particle is removed from the population of virus that maintains the ability to infect additional uninfected cells. However, viral cytopathic effects are insignificant when assessing the overall elimination of viral particles and thus, are not illustrated in the model [2]. Immune-mediated cellular destruction is the immune system's ability to clear, or eliminate, the virus from the body. This method typically eliminates a majority of the virus population [4]. The model assumes that the virus is then killed off at a clearance rate, $\gamma$, proportional to the virus population $[16]$.

Equation 1d highlights the dynamics of CTLs. The CTL population is composed of production rate and death rate. The overall equation is:

$$
\text { Rate of change of CTL population }=(\text { Production rate })-(\text { Death rate })
$$

Production rate: CTLs are activated when contact between the CTL and an infected cell is made within the infected host. Thus, the production rate, $\rho$, can be assumed to be proportional to the product of the CTL and infected cell populations.

Death rate: Similar to uninfected cell death, CTLs are cleared by the immune system at a rate, $\delta$, proportional to the CTL population.

It is important to note that all of the model parameters are presumed to be positive. In addition, there are two biologically reasonable assumptions we are able to make with regard to the values of parameters in relation to one another. Notably, it is biologically reasonable to assume that infected cells have a higher death rate than uninfected cells, namely $\alpha \geq \mu$. Furthermore, we are also able to assume that the death rate of infected cells is greater than the natural death rate of CTLs, and thus $\alpha \geq \delta$.

\section{Analysis of the Model}

\subsection{Existence and Uniqueness of Solutions}

Prior to conducting an in-depth analysis of the model, it is crucial to show that the solutions to the initial-value problem exist and are unique.

\subsection{Positivity and Boundedness}

In order to retain the biological validity of the model, we must prove that solutions to the system of differential equations are positive and bounded for all values of time. For example, concluding that a population 


\section{ANALYSIS OF THE MODEL}

is negative is not biologically feasible. Furthermore, the populations must remain finite since the human body can only be composed of a finite number of cells. In addition, boundedness and positivity illustrate that once infected, it is possible that the population of the virus will continue to exist beneath the detectable threshold without doing significant damage [21]. The next step in analyzing our model will be to prove positivity and boundedness for the system of differential equations. We will do so by proving the following theorems.

Lemma (Positivity). Let $t_{0}>0$. In the model, if the initial conditions satisfy $X(0)>0, I(0)>0, V(0)>0$, $T(0)>0$ then for all $t \in\left[0, t_{0}\right], X(t), I(t), V(t), T(t)$ will remain positive in $\mathbb{R}_{+}^{4}$.

Proof: Positivity. We must prove that for all $t \in\left[0, t_{0}\right], X(t), I(t), V(t), T(t)$ will be positive in $\mathbb{R}_{+}^{4}$. We know that all of the parameters used in the system are positive. Thus, we can place lower bounds on each of the equations given in the model. Thus,

$$
\begin{gathered}
\frac{d X}{d t}=\lambda-\mu X(t)-\beta V(t) X(t) \geq-\mu X(t)-\beta V(t) X(t) \\
\frac{d I}{d t}=\beta V(t) X(t)-\alpha I(t)-\rho I(t) T(t) \geq-\alpha I(t)-\rho I(t) T(t) \\
\frac{d V}{d t}=c Y(t)-\gamma V(t) \geq-\gamma V(t) \\
\frac{d T}{d t}=\rho Y(t) T(t)-\delta T(t) \geq-\delta T(t)
\end{gathered}
$$

Through basic differential equations methods we can resolve the inequalities and produce:

$$
\begin{gathered}
X(t) \geq e^{-\mu t-\beta \int V(t) d t} \geq 0 \\
I(t) \geq e^{-\alpha t-\rho \int T(t) d t} \geq 0 \\
V(t) \geq e^{-\gamma t} \geq 0 \\
T(t) \geq e^{-\delta t} \geq 0
\end{gathered}
$$

Thus, for all $t \in\left[0, t_{0}\right], X(t), I(t), V(t), T(t)$ will be positive and remain in $\mathbb{R}_{+}^{4}$.

Lemma (Boundedness). There exists an $X_{M}, I_{M}, V_{M}, T_{M}>0$ such that for $X(t), I(t), I(t), T(t) \limsup _{t \rightarrow \infty}(X(t)) \leq$ $X_{M}, \lim \sup _{t \rightarrow \infty}(I(t)) \leq I_{M}, \lim \sup _{t \rightarrow \infty}(V(t)) \leq V_{M}, \limsup _{t \rightarrow \infty}(T(t)) \leq T_{M}$ for all $t \in\left[0, t_{0}\right]$.

Proof: Boundedness. We must prove that for all $t \in\left[0, t_{0}\right], X(t), I(t), V(t), T(t)$ will be bounded. We know that all of the constants used in the system are positive.

$$
\frac{d X}{d t}+\frac{d I}{d t}+\frac{d T}{d t}=\lambda-\mu X(t)-\alpha I(t)-\delta T(t)
$$

Since all of the constants are positive,

$$
\frac{d(X+I+T)}{d t} \leq \lambda-\min \{\mu, \alpha, \delta\}(X+I+T)(t)
$$

which implies,

$$
(X+I+T)(t) \leq \frac{\lambda}{\min \{\mu, \alpha, \delta\}}+c_{0} e^{-\min \{\mu, \alpha, \delta\} t}
$$

taking the limsup of both sides,

$$
\limsup _{t \rightarrow \infty}(X+I+T)(t) \leq \limsup _{t \rightarrow \infty}\left(\frac{\lambda}{\min \{\mu, \alpha, \delta\}}+c_{0} e^{-\min \{\mu, \alpha, \delta\} t}\right)=\frac{\lambda}{\min \{\mu, \alpha, \delta\}}
$$

So, choose

$$
X_{M}=I_{M}=T_{M}=\frac{\lambda}{\min \{\mu, \alpha, \delta\}}
$$

Thus, $(X+I+T)(t)$ is bounded, so $X(t), I(t)$, and $T(t)$ are all bounded since 


\section{ANALYSIS OF THE MODEL}

$$
X(t), I(t), T(t) \leq(X+I+T)(t) .
$$

So,

$$
X(t) \leq X_{M}, I(t) \leq I_{M}, \text { and } T(t) \leq T_{M} \text { for all } t \in\left[0, t_{0}\right]
$$

Furthermore, since all of the constants are positive, we can place an upper bound on $\frac{d V}{d t}$ so,

$$
\frac{d V}{d t}=c I(t)-\gamma V(t) \leq c I(t)
$$

Therefore, we can choose

$$
V_{M}=c I_{M}
$$

Thus,

$$
V(t) \leq c I_{M}=V_{M}
$$

Hence, since $I(t)$ is bounded for all $t \in\left[0, t_{0}\right]$, we know that $V(t)$ is bounded for all $t \in\left[0, t_{0}\right]$.

Theorem 1 (Existence). Let $t_{0}>0$. In the model, if the initial conditions satisfy $X(0)>0, I(0)>0, V(0)>0$, $T(0)>0$ then $\forall t \in \mathbb{R} X(t), I(t), V(t), T(t)$ will exist in $\mathbb{R}_{+}^{4}$.

Proof: Existence and Uniqueness. In the case of our model we have:

$$
\mathbf{x}=\left[\begin{array}{c}
X(t) \\
I(t) \\
V(t) \\
T(t)
\end{array}\right] \text { and } \mathbf{f}(\mathbf{x})=\left[\begin{array}{c}
\lambda-\mu X(t)-\beta V(t) X(t) \\
\beta V(t) X(t)-\alpha I(t)-\rho I(t) T(t) \\
c I(t)-\gamma V(t) \\
\rho I(t) T(t)-\delta T(t)
\end{array}\right]
$$

Note that $f$ has a continuous derivative on $\mathbb{R}^{4}$ and thus, $f$ is locally Lipschitz in $\mathbb{R}^{4}$. Hence, by the Fundamental Existence and Uniqueness Theorem located in the appendix as well as the lemmas proved on positivity and boundedness of solutions, we know that there exists a unique, positive, and bounded solution to the ordinary differential equations given in $1(a)-1(d)$.

\subsection{Equilibria}

For the model we consider the equilbiria for the populations $(X, I, V, T)$. At the equilibrium, the rate of change for each population is zero. Thus, we obtain these values by setting each differential equation simultaneously equal to zero. Therefore, $\frac{d X}{d t}=0, \frac{d I}{d t}=0, \frac{d V}{d t}=0, \frac{d T}{d t}=0$.

From a biological perspective we are able to classify these equilibria as viral persistence equilibrium or viral free equilibrium. If the values for any population at the equilibrium is zero $(X=0, I=0, V=0, T=0)$, those cells are defined as extinct, meaning that as $t \rightarrow \infty$ the populations will become zero. Thus, if $V=I=0$ at the equilibrium, the virus is extinct from the body as $t \rightarrow \infty$ and the equilibrium is known as a viral free equilibrium (also referred to as an infection free steady state). However, if the value for any population at the equilibrium is not zero $(X \neq 0, I \neq 0, V \neq 0, T \neq 0)$ those cells are defined as persistent. Thus, if $V \neq 0$ and $I \neq 0$, then the virus persists and the equilibrium is known as a viral persistence equilibrium (also known as a chronic infection steady state). In addition, if $T \neq 0$, then the immune response persists as $t \rightarrow \infty$ and we classify the equilibrium as an immune persistence equilibrium.

If the system takes on an equilibrium at any time, it will remain at the value for all remaining time; however, unless the initial conditions are exactly one of the equilibria, the system need not necessarily obtain these values. The system may approach the equilibrium, move away from the equilibrium, or cycle between specific equilibria. In order to accurately determine which type of behavior the system will yield, we must perform a stability analysis for the system. 


\section{APPLICATION TO THE MODEL}

\subsection{Linearization and the Jacobian}

In modeling systems it becomes apparent that nearly all systems are non-linear, including the model we are examining. However, most of the theory that has been developed by mathematicians governing the behavior of systems of differential equations, especially stability, is centered upon linear systems. Thus, in order to further understand the behavior of a non-linear system it is first crucial to linearize the system. Essentially, this process approximates a non-linear system in a linear manner near the values around which a linear approximation occurs. The linear approximation occurs at the equilibria, which will be denoted $P_{n}$.

In a neighborhood of the equilibria we can make a linear approximation and so determine the local character of the paths. This technique allows the stability of the system at the equilibria to be determined and provides a starting point for global investigations of solutions. The goal of this stability analysis is to perturb the system from equilibrium and study the behavior of the system. Thus, we will look to see if the solutions move towards or away from equilibrium.

In order to linearize the system, we must compute the Jacobian matrix of the system. The Jacobian is the matrix of the partial derivatives of each function with respect to each variable. Essentially, the Jacobian provides a linear approximation of a system at any given value.

\subsection{Local Stability}

Analysis of the eigenvalues of the Jacobian matrix evaluated at the equilibrium gives insights into the local stability properties at that equilibrium. We will apply the Poincare-Perron Theorem, Routh-Hurwitz Criteria, and the Hartman-Grobman Theorem, which can all be found in the Appendix, as well as analyze the eigenvalues to determine the local asymptotic stability properties of our system of differential equations.

\section{Application to the Model}

The model has three biologically relevant equilibria denoted $P_{n}=(X, I, V, T)$ for $n=1,2,3$ :

$$
\begin{aligned}
& P_{1}=\left(\frac{\lambda}{\mu}, 0,0,0\right) \\
& P_{2}=\left(\frac{\alpha \gamma}{c \beta}, \frac{c \beta \lambda-\alpha \gamma \mu}{c \alpha \beta}, \frac{c \beta \lambda-\alpha \gamma \mu}{\alpha \beta \gamma}, 0\right) \\
& P_{3}=\left(\frac{\gamma \lambda \rho}{c \beta \delta+\gamma \mu \rho}, \frac{\delta}{\rho}, \frac{c \delta}{\gamma \rho}, \frac{-c \alpha \beta \gamma+c \beta \lambda \rho-\alpha \gamma \rho \mu}{\rho(c \beta \delta+\gamma \mu \rho)}\right)
\end{aligned}
$$

We characterize $P_{1}$ as a viral free equilibrium; this means that as $t \rightarrow \infty$, the virus will be eliminated from the body. $P_{2}$ and $P_{3}$ describe the persistence of the virus, and thus can be characterized as a viral persistence equilibrium. At these values, the virus will remain in the system as $t \rightarrow \infty$. The equilibrium also describe the persistence of an immune response. We denote that at $P_{2}$ and $P_{1}$ the immune response is either not required, or may even be suppressed as $t \rightarrow \infty$, while at $P_{3}$ the immune system's response remains present for all time, even after the virus population reaches a stable level. Notably, $P_{3}$ is the only equilibrium which describes the co-existence of virus and CTLs as $t \rightarrow \infty$. In addition, it is important to denote that all of the equilibria have a persistence of uninfected cells.

\subsection{Jacobian}

The Jacobian for the linearized system is:

$$
J(X, I, V, T)=\left[\begin{array}{cccc}
V \beta-\mu & 0 & -X \beta & 0 \\
V \beta & \alpha-T \rho & X \beta & -I \rho \\
0 & c & -\gamma & 0 \\
0 & T \rho & 0 & -\delta+I \rho
\end{array}\right]
$$




\section{APPLICATION TO THE MODEL}

The characteristic polynomial is defined as the polynomial side of the characterstic equation, $\operatorname{det}(A-\lambda I)=0$ where $A$ is a square matrix, $I$ is the identity matrix, and $\lambda$ is an eigenvalue. The roots of the characteristic polynomial of the Jacobian will tend to depend on several parameters known as threshold parameters. The values of these parameters, sometimes called the reproductive constants, influence and determine the stability of the system.

Define:

$$
\begin{aligned}
& R_{0}=\frac{c \beta \lambda}{\alpha \gamma \mu} \\
& R_{1}=\frac{c \beta \lambda \rho}{\alpha(\gamma \rho \mu+c \beta \delta)}
\end{aligned}
$$

to be the reproductive constants of the system. Biologically, $R_{0}$ represents the average number of infected cells produced by an initially infected cell over its lifetime [6]. The value of $R_{0}$ is a well established norm when discussing viral infections [6]. The $R_{0}$ value associated with Ebola is generally accepted to range from 2 to 3 [1]. This value depends upon the parameters of the individual who is infected, but also varies based on the geographical location of the disease. $R_{1}$ translates the notion of viral reproductive constants to the immune system response. Thus, $R_{1}$ represents the number of infected cells that a single immune cell (CTL in the case of our model) is able to address. The interpretation of $R_{1}$ will be discussed in greater detail in Section 4.5.

Three theorems will be presented to highlight the relationship between the two reproductive constants and the local asymptotic stability of the equilibria. Thus, we are able to examine the values of $R_{0}, R_{1}$ and a few other simple expressions to determine whether viral persistence or viral extinction occurs as $t \rightarrow \infty$. As a result, we may be able to predict the persistence of the Ebola virus upon initial infection simply by determining the values of these expressions. In addition, we will also be able to determine whether or not the immune response is suppressed during infection.

\subsection{Stability Analysis for $P_{1}$}

The Jacobian evaluated at $P_{1}=\left(\frac{\lambda}{\mu}, 0,0,0\right)$ becomes:

$$
J_{1}=\left[\begin{array}{cccc}
-\mu & 0 & -\frac{\beta \lambda}{\mu} & 0 \\
0 & \alpha & \frac{\beta \lambda}{\mu} & 0 \\
0 & c & -\gamma & 0 \\
0 & 0 & 0 & -\delta
\end{array}\right]
$$

Furthermore, the characteristic equation for $P_{1}$ is

From the characteristic equation we can define:

$$
-\frac{1}{\mu}(-x-\delta)(x+\mu)\left(-c \beta \lambda+x^{2} \mu+x \alpha \mu+x \gamma \mu+\alpha \gamma \mu\right)=0
$$

$$
\begin{aligned}
& a_{1}=\alpha+\gamma+\delta+\mu \\
& a_{2}=\alpha(\gamma+\delta+\mu)-\frac{\beta c \lambda}{\mu}+\gamma(\delta+\mu)+\delta \mu \\
& a_{3}=\frac{\mu(\alpha(\gamma(\delta+\mu)+\delta \mu)+\gamma \delta \mu)-\beta c \lambda(\delta+\mu)}{\mu} \\
& a_{4}=\alpha \gamma \delta \mu-\beta c \delta \lambda^{\mu}
\end{aligned}
$$

such that $-\frac{1}{\mu}(-x-\delta)(x+\mu)\left(-c \beta \lambda+x^{2} \mu+x \alpha \mu+x \gamma \mu+\alpha \gamma \mu\right)=x^{4}+a_{1} x^{3}+a_{2} x^{2}+a_{3} x+a_{4}$.

\subsubsection{Analysis:}

Theorem 2 (Local Asymptotic Stability of $\left.P_{1}\right)$. For the viral extinction equilibrium $\left(P_{1}\right)$ given by

$$
(X, I, V, T)=\left(\frac{\lambda}{\mu}, 0,0,0\right)
$$

if $R_{0}<1$, then $P_{1}$ is stable; however if $R_{0}>1$, then $P_{1}$ is unstable. 


\section{APPLICATION TO THE MODEL}

Proof. We will use the Routh-Hurwitz Criteria and the values of $a_{1}, a_{2}, a_{3}$, and $a_{4}$ to derive the stability of $P_{1}$. We know that all of the parameters are positive. Therefore, $a_{1}$ is clearly $>0$.

We can write $a_{2}$ as,

$$
a_{2}=\left(1-R_{0}\right) \frac{1}{\alpha \gamma \mu}\left((\alpha+\gamma) \mu(\delta+\mu)+\delta \mu^{2}\right) .
$$

Thus, if $R_{0}<1$, then $a_{2}>0$ since all the parameters are positive. However, if $R_{0}>1$, then clearly $a_{2}<0$.

Furthermore, $a_{3}$ can be represented by,

$$
a_{3}=\left(1-R_{0}\right)(\delta+\mu) \frac{1}{\alpha \delta \mu}(\delta \mu(\alpha+\gamma))
$$

Thus, if $R_{0}<1$, then $a_{3}>0$; however, if $R_{0}>1$, then $a_{3}<0$.

In addition,

$$
a_{4}=\left(1-R_{0}\right) \frac{\delta}{\alpha \gamma \mu}
$$

Thus, if $R_{0}<1$, then $a_{4}>0$; however, if $R_{0}>1$, then $a_{4}<0$.

Finally,

$$
a_{1} a_{2} a_{3}-\left(a_{3}^{2}+a_{1}^{2} a_{4}\right)=\frac{1}{\mu^{2}}\left((\alpha+\gamma)(\delta+\mu)\left(1-R_{0}+\alpha \delta \mu+\gamma \delta \mu+\delta^{2} \mu\right)\left(1-R_{0}+\alpha \mu^{2}+\gamma \mu^{2}+\mu^{3}\right)\right)
$$

Thus, if $R_{0}<1$, then $a_{1} a_{2} a_{3}-\left(a_{3}^{2}+a_{1}^{2} a_{4}\right)>0$. and therefore, $a_{1} a_{2} a_{3}>a_{3}^{2}+a_{1}^{2} a_{4}$. Thus, if $R_{0}<1$ all of the conditions necessary for stability are met and $P_{1}$ is stable. However, if $R_{0}>1$, then $a_{2}, a_{3}, a_{4}<0$ and $P_{1}$ is unstable.

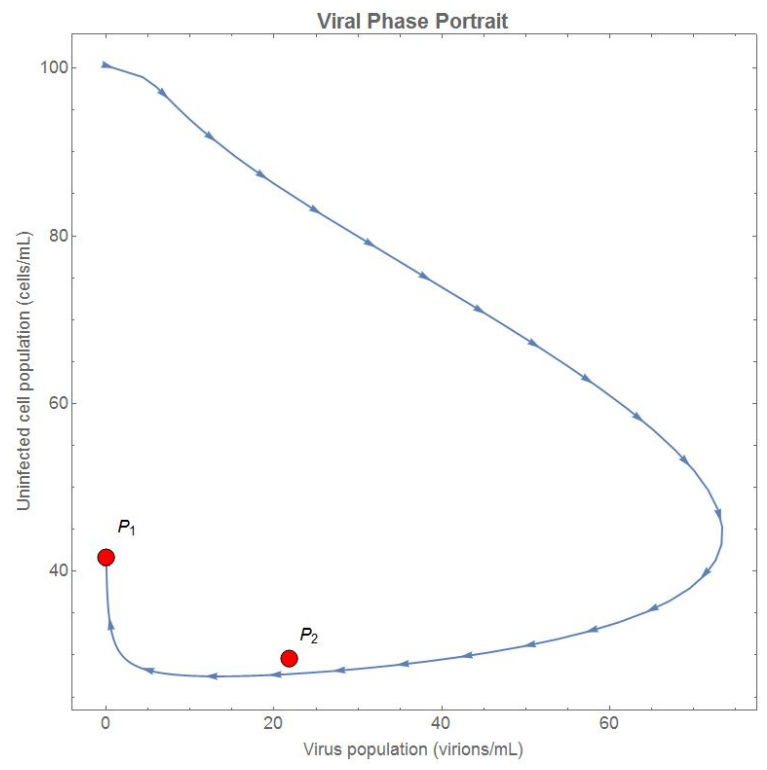

Figure 2: The viral phase portrait for $R_{0}=0.9373$ and $R_{1}=0.5207$.

highlights the local asymptotic stability of the two populations at the equilibrium.
Figure 2 illustrates the interaction between the virus and uninfected cell populations given that $R_{0}, R_{1}<1$. The arrows in the graph illustrate the progressions of the viral and uninfected populations relative to one another over time. Figure 2 reveals that upon introduction of the virus, the population of uninfected cells decreases relative to the viral population. This behavior continues until the virus reaches the peak viral load point, or the maximum value for the virus population. After reaching this value, the virus population begins to decline until it reaches zero. This suggests that either the immune system has the ability to suppress the increase in viral load, or that the virus does not maintain an infection rate which is high enough in order to sustain chronic infection, i.e. ensuring its persistence.

Based on our analysis in the previous section as well as the values for $R_{0}$ and $R_{1}$, we expect that the equilibrium will be locally asymptotically stable and that the equilibrium should be an infection-free equilibrium. Figure 2 reveals that as $t \rightarrow \infty$ the viral load goes to zero, thereby suggesting that the equilibrium is in fact a viral extinction equilibrium. This is precisely the behavior that we expected given the values of $R_{0}$ and $R_{1}$. Thus, Figure 2 


\section{APPLICATION TO THE MODEL}

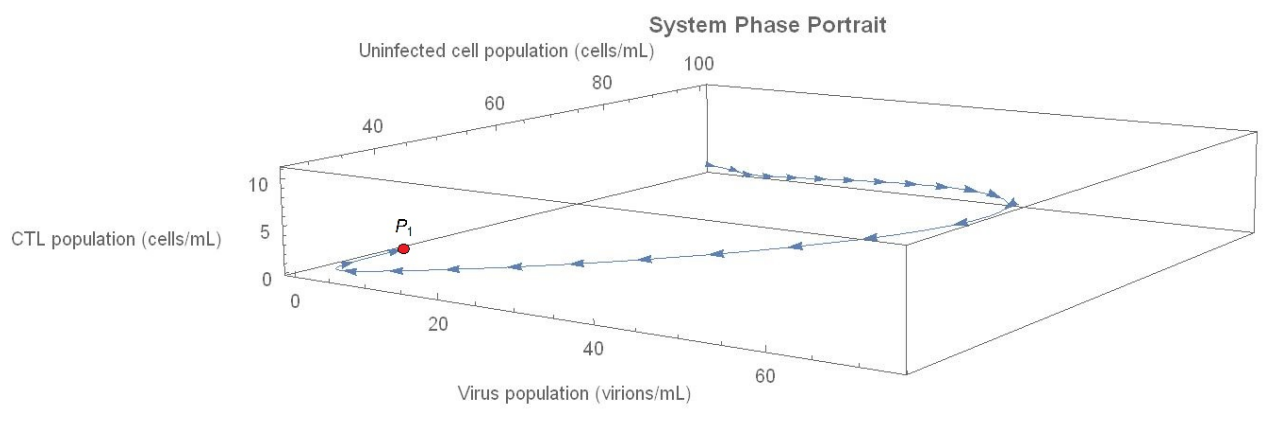

Figure 3: A system phase portrait for $R_{0}=0.9373$ and $R_{1}=0.5207$.

Figure 3 describes the dynamic between the virus and uninfected cell populations; however, this figure underscores the interactions of both of these populations while also considering the population of CTLs. Thus, Figure 3 illustrates the dynamic of the virus and uninfected cells relative to a primary actor in an immune response. In addition, Figure 3 highlights the local stability of the system. The figure suggests that the viral extinction equilibrium is locally asymptotically stable which is exactly what we expect given the values of $R_{0}$ and $R_{1}$.

In addition, Figure 3 suggests that the CTL response raised by the immune system is sufficient to control the spread and proliferation of the virus. However, the figure also reveals that the population of CTLs is relatively low when compared with both the viral load as well as the population of uninfected cells. Thus, it appears as though the immune system did not need to mount an extremely robust response in order to stop the increase in viral load. Furthermore, Figure 3 illustrates that as $t \rightarrow \infty$ the CTL population diminishes and becomes zero, which corresponds with our biological understanding of immune system function post infection; this is expected since the virus is eliminated from the

CTL population (cells $/ \mathrm{mL}$ )

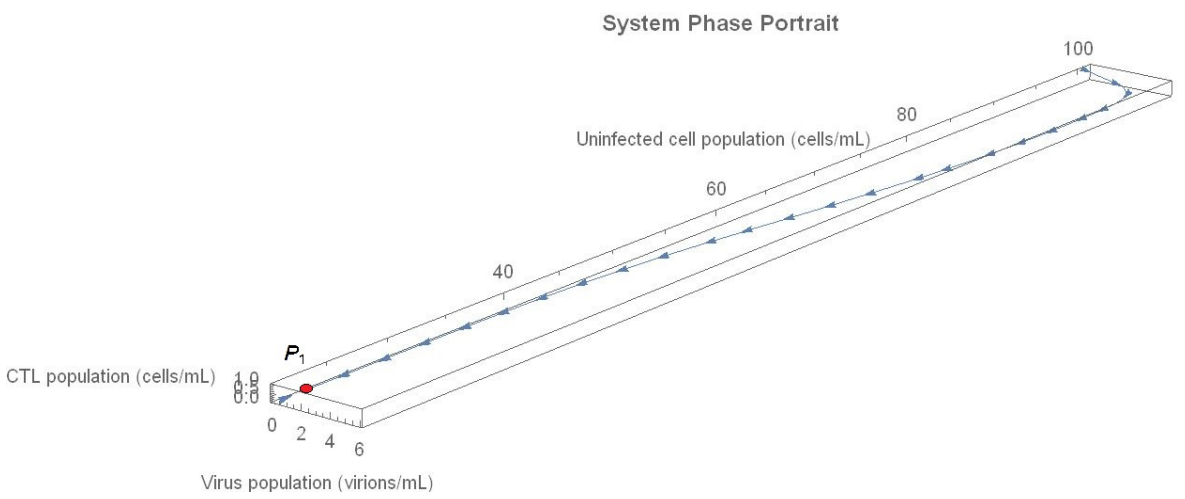

Figure 4: The system phase portrait for $R_{0}=0.2464$ and $R_{1}=0.1760$. immune system during a successful immune challenge.

Figure 4 highlights the system interaction between the virus, uninfected cell, and CTL populations given that $R_{0}, R_{1}<1$. Figure 4 again reveals that the equilibrium is locally stable since the trajectory approaches $P_{1}$ and that the virus population as $t \rightarrow \infty$ is equal to zero, thereby suggesting that the equilibrium is a viral extinction equilibirum. Furthermore, Figure 4 highlights that the immune response was extremely small in magnitude when compared to Figure 3.

Thus, it appears as though the virus does not reach a high enough level to either stimulate a robust immune response or escape any immune response since the both the population of virus and CTL population remain relatively low. This suggests that the magnitude of $R_{0}$ and $R_{1}$ may reveal the magnitude of the immune response. In addition, it suggests that the low infection rate means that the population of virus will likely be controlled solely via natural death. 


\section{APPLICATION TO THE MODEL}

\subsection{Stability Analysis for $P_{2}$}

The Jacobian evaluated at $P_{2}=\left(\frac{\alpha \gamma}{c \beta}, \frac{c \beta \lambda-\alpha \gamma \mu}{c \alpha \beta}, \frac{c \beta \lambda-\alpha \gamma \mu}{\alpha \beta \gamma}, 0\right)$ is:

$$
J_{2}=\left[\begin{array}{cccc}
-\mu-\frac{c \beta \lambda-\alpha \gamma \mu}{\alpha \gamma} & 0 & -\frac{\alpha \lambda}{c} & 0 \\
\frac{c \beta \lambda-\alpha \gamma \mu}{\alpha \gamma} & \alpha & \frac{\alpha \lambda}{c} & -\frac{(c \beta \lambda-\alpha \gamma \mu) \rho}{c \alpha \beta} \\
0 & c & -\gamma & 0 \\
0 & 0 & 0 & -\delta+\frac{(c \beta \lambda-\alpha \gamma \mu) \rho}{c \alpha \beta}
\end{array}\right]
$$

Furthermore, the characteristic equation for $P_{2}$ is

$$
-\frac{1}{c^{2} \alpha^{2} \beta \gamma^{2}}\left(c^{2} \alpha(x+\alpha) \beta(-x-\gamma) \gamma(x \alpha \gamma+c \beta \lambda)-c\left(-c x \alpha^{3} \beta \gamma^{3}-c \alpha^{3} \beta \gamma^{3} \mu\right)\right)\left(-x-\delta+\frac{(c \beta \lambda-\alpha \gamma \mu) \rho}{c \alpha \beta}\right)=0
$$

\subsubsection{Analysis:}

Theorem 3 (Local Asymptotic Stability of $P_{2}$ ). For the viral persistence equilibrium and immune suppressive equilibirum $\left(P_{2}\right)$ given by

$$
(X, I, V, T)=\left(\frac{\alpha \gamma}{c \beta}, \frac{c \beta \lambda-\alpha \gamma \mu}{c \alpha \beta}, \frac{c \beta \lambda-\alpha \gamma \mu}{\alpha \beta \gamma}, 0\right)
$$

$P_{2}$ is stable iff $R_{0}>1$ and $R_{1}<1$.

Proof. One eigenvalue of $J_{2}$ is $x_{1}=\frac{c \beta \lambda \rho-(c \alpha \beta \delta+\alpha \gamma \mu \rho)}{c \alpha \beta}$.

Thus, we can simplify the characteristic equation. As a result the remaining two eigenvalues are solutions to the equation,

$$
x^{3}+x^{2} \frac{\alpha^{2} \gamma+\alpha \gamma^{2}+c \beta \lambda}{\alpha \gamma}+x \frac{c \beta(\alpha+\gamma)}{\alpha \gamma}+c \beta \lambda-\alpha \gamma \mu=0
$$

We will then use the Routh-Hurwitz criteria to prove that all of the roots are negative given $R_{0}>1$ and $R_{1}<1$. From the characteristic equation we obtain,

$$
\begin{aligned}
& a_{1}=\frac{\alpha^{2} \gamma+\alpha \gamma^{2}+c \beta \lambda}{\alpha \gamma} \\
& a_{2}=\frac{c \beta(\alpha+\gamma)}{\alpha \gamma} \\
& a_{3}=c \beta \lambda-\alpha \gamma \mu
\end{aligned}
$$

We know $a_{1}, a_{2}>0$ since all of the parameters are positive.

Furthermore, we can denote:

$$
a_{3}=c \beta \lambda-\alpha \gamma \mu=\left(R_{0}-1\right) \frac{1}{\alpha \gamma \mu}
$$

Thus, if $R_{0}>1$ then $a_{3}>0$, however, if $R_{0}<1$ then $a_{3}<0$ and $P_{2}$ is unstable.

In addition, we can define:

$$
a_{1} a_{2}-a_{3}=c \beta \lambda+\frac{c \beta \alpha \lambda}{\gamma}+\frac{c \beta \gamma \lambda}{\alpha}+\frac{c^{2} \beta^{2} \lambda^{2}}{\alpha \gamma^{2}}+\frac{c^{2} \beta^{2} \lambda^{2}}{\alpha^{2} \gamma}+\alpha \gamma \mu
$$

Also, since all of the parameter are positive $a_{1} a_{2}-a_{3}>0$ which implies that $a_{1} a_{2}>a_{3}$. 


\section{APPLICATION TO THE MODEL}

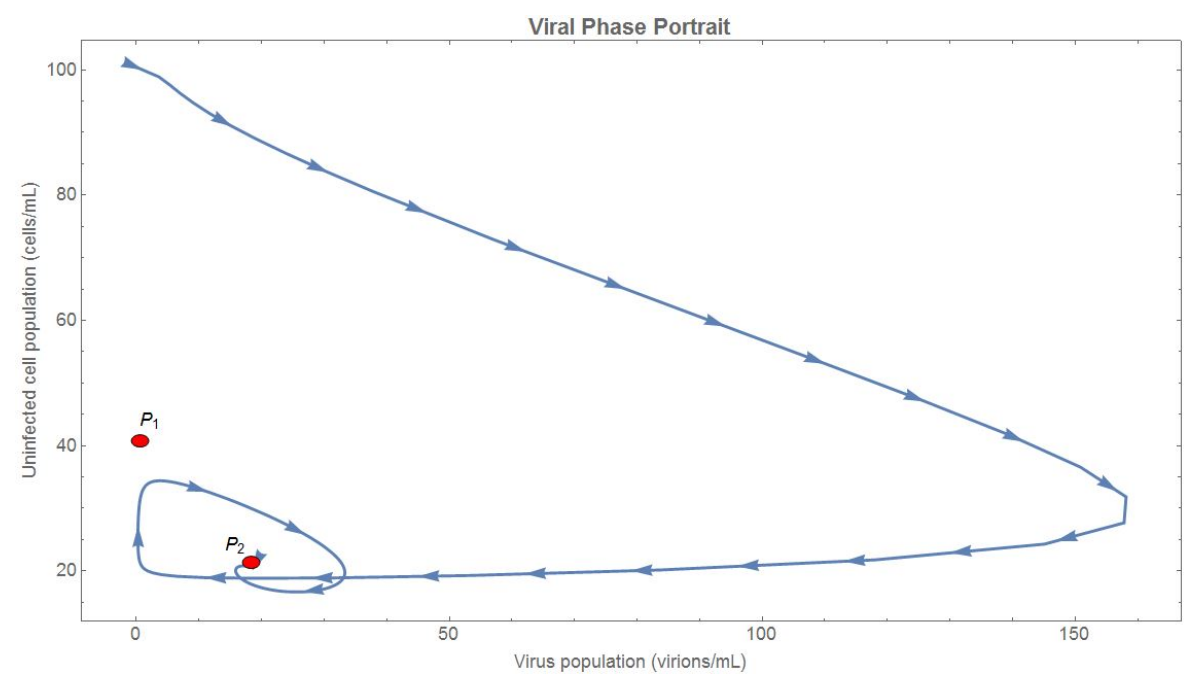

Figure 5: A viral phase portrait for $R_{0}=2.1089$ and $R_{1}=0.84356$.
Figure 5 illustrates the dynamic between the virus and uninfected cell populations given the values $R_{0}>1$ and $R_{1}<1$. In this case, $P_{1}=(42.2,0,0,0)$ and $P_{2}=$ $(20,3.70,18.48,0)$. Upon introduction of the virus, the population of uninfected cells begins to decrease relative to the population of the virus until the virus population reaches the peak viral load point. After this point, the population of the uninfected cells remains relatively constant whereas there is a decrease in the viral load. In terms of stability, Figure 5 demonstrates the persistence of

the virus since the population of the virus remains positive as $t \rightarrow \infty$ and the equilibrium is locally asymptotically stable.

The introduction of virus clearly has a large impact on the magnitude of the uninfected cell population. Throughout the course of infection, the uninfected cell population is reduced to $20 \%$ of it's original size. This may be associated with a loss of functionality of the cells the virus infects, and may prove to be deleterious for an infected individual. However, it also appears as though the viral load reaches extremely low levels as the population of virus approaches the equilibrium. This behavior may be consistent with a suppression of the virus population by the immune response. However, this behavior might also suggest the presence of a viral latency period in which the virus population remains dormant at very low levels within the immune system. During such a stage, the immune system would not have the ability to detect the presence of the virus, and thus would not be able to mount an effective response. In addition, Figure 5 illustrates the resurgence of the virus after a possible latency stage; it appears as though the virus is able to re-infect the host since the virus population increases as the trajectory approaches the stable node.

Figure 6 underscores the dynamic between the virus and immune response. From this figure we can see that the population of CTLs does not significantly increase until the virus reaches the peak viral load. Thus,

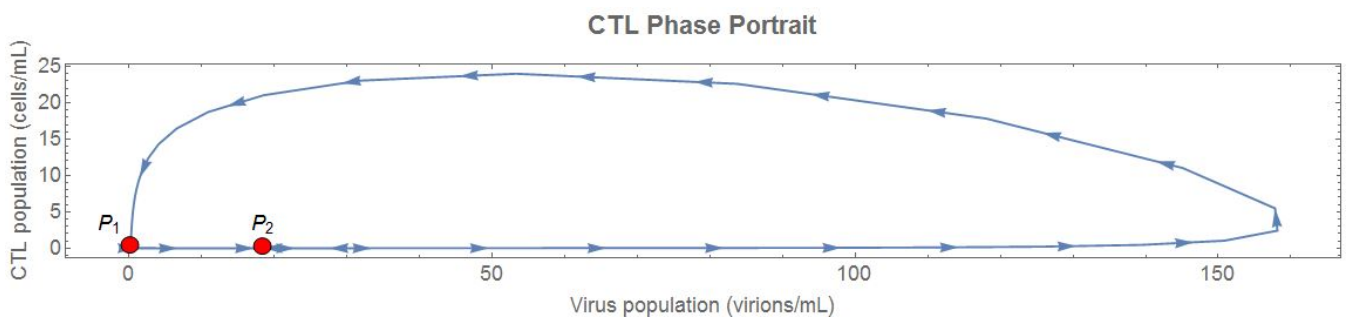
the figure suggests

the presence of a CTL lag phase. This type of behavior is regular of a natural immune response. In addition, Figure 6 suggests that the viral load increases at a rate which is significantly greater than the rate at which the CTLs recognize the virus, activate, and proliferate. Yet, even though an immune response is activated upon the virus reaching the peak viral load, the immune response, as reflected by the CTL population, ultimately deteriorates and is unable to address and stop the proliferation and eventual persistence of the virus. Thus, the virus successfully "evades" the immune response. 


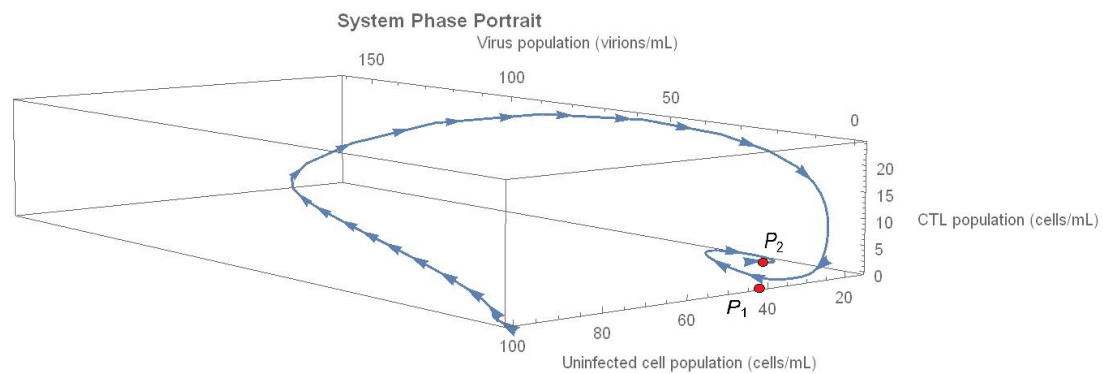

Figure 7: A system phase portrait for $R_{0}=2.1089$ and $R_{1}=0.84356$.
Figure 7 shows the dynamic between the virus and uninfected cell populations, however Figure 7 integrates both Figure 5 and Figure 6 to highlight the interactions of the viral and uninfected populations with the CTL population developed during the immune response. Again, Figure 7 highlights the local stability of the system, as well as the persistence of the virus which is exactly what we expect given the values of $R_{0}$ and $R_{1}$. The figure also illustrates that as $t \rightarrow \infty$ the immune response, as reflected by the CTL population, fades and becomes zero. Thus, the immune system is ineffective at controlling the increase in viral load and the individual remains chronically infected.

\subsection{Stability Analysis for $P_{3}$}

The Jacobian evaluated at $P_{3}=\left(\frac{\gamma \lambda \rho}{c \beta \delta+\gamma \mu \rho}, \frac{\delta}{\rho}, \frac{c \delta}{\gamma \rho}, \frac{-c \alpha \beta \gamma+c \beta \lambda \rho-\alpha \gamma \rho \mu}{\rho(c \beta \delta+\gamma \mu \rho)}\right)$ is:

$$
J_{3}=\left[\begin{array}{cccc}
-\mu-\frac{c \beta \delta}{\rho \gamma} & 0 & -\frac{\beta \gamma \lambda \rho}{c \beta \delta+\gamma \rho \mu} & 0 \\
\frac{c \beta \delta}{\rho \gamma} & \alpha-\frac{-c \alpha \beta \delta+c \beta \lambda \rho-\alpha \gamma \mu \rho}{c \beta \delta+\gamma \mu \rho} & \frac{\beta \gamma \lambda \rho}{c \beta \delta+\gamma \mu \rho} & -\delta \\
0 & c & -\gamma & 0 \\
0 & \frac{-c \alpha \beta \delta+c \beta \lambda \rho-\alpha \gamma \mu \rho}{c \beta \delta+\gamma \rho \mu} & 0 & 0
\end{array}\right]
$$

Furthermore, the characteristic equation for $P_{3}$ is

$$
-\frac{x\left(\beta c \gamma^{2} \lambda \rho^{2}(\mu+x)-(\alpha+x)(\gamma+x)(\beta c \delta+\gamma \mu \rho)(\beta c \delta+\gamma \rho(\mu+x))\right)+\rho \mathrm{Y}(\gamma+x)(\beta c \delta+\gamma \rho(\mu+x))(\alpha \gamma \mu \rho+\beta c(\alpha \delta-\lambda \rho))}{\gamma \rho(\beta c \delta+\gamma \mu \rho)}=0
$$

Therefore,

$$
\begin{aligned}
& a_{1}=\frac{\beta^{2} c^{2} \delta^{2}+\gamma^{2} \mu \rho^{2}(\gamma+\mu)+\beta c \gamma \rho(\gamma \delta+2 \delta \mu+\lambda \rho)}{\gamma \rho(\beta c \delta+\gamma \mu \rho)} \\
& a_{2}=\frac{\gamma^{2} \mu \rho^{2}(\gamma \mu-\alpha \delta)+\beta^{2} c^{2} \delta(\gamma \delta+\lambda \rho)+\beta c \gamma \rho\left(-\alpha \delta^{2}+2 \gamma \delta \mu+\lambda \rho(\delta+\mu)\right)}{\gamma \rho(\beta c \delta+\gamma \mu \rho)} \\
& a_{3}=\frac{\delta\left(-\alpha \gamma^{2} \mu \rho^{2}(\gamma+\mu)+\beta^{2} c^{2}\left(\lambda \rho(\gamma+\delta)-\alpha \delta^{2}\right)+\beta c \gamma \rho(\lambda \rho(\gamma+\mu)-\alpha \delta(\gamma+2 \mu))\right)}{\gamma \rho(\beta c \delta+\gamma \mu \rho)} \\
& a_{4}=\delta\left(\beta c\left(\lambda-\frac{\alpha \delta}{\rho}\right)-\alpha \gamma \mu\right)^{\gamma(\beta)}
\end{aligned}
$$

\subsubsection{Analysis:}

Theorem 4 (Local Asymptotic Stability of $P_{3}$ ). For the viral persistence equilbirium and immune response persistence equilbirium $\left(P_{3}\right)$ given by

$$
(X, I, V, T)=\left(\frac{\gamma \lambda \rho}{c \beta \delta+\gamma \mu \rho}, \frac{\delta}{\rho}, \frac{c \delta}{\gamma \rho}, \frac{-c \alpha \beta \gamma+c \beta \lambda \rho-\alpha \gamma \rho \mu}{\rho(c \beta \delta+\gamma \mu \rho)}\right)
$$

If $R_{1}>1, R_{0}>\frac{\delta}{\mu}, c \beta>\alpha \rho, \lambda(\gamma+\delta)>\alpha \delta^{2}$ then $P_{3}$ is stable.

Proof. We know that since all the parameters are positive, $a_{1}>0$.

Taking the numerator of $a_{2}$, we want $\delta\left(-\alpha \gamma^{2} \mu \rho^{2}(\gamma+\mu)+\beta^{2} c^{2}\left(\lambda \rho(\gamma+\delta)-\alpha \delta^{2}\right)+\beta c \gamma \rho(\lambda \rho(\gamma+\mu)-\alpha \delta(\gamma+2 \mu))\right)>0$. Thus, if $\gamma^{3} \mu^{2} \rho^{2}+\beta^{2} c^{2} \delta(\gamma \delta+\lambda \rho)+\beta c \gamma \rho(2 \gamma \delta \mu+\lambda \rho(\delta+\mu))>\alpha \gamma \delta \rho(\beta c \delta+\gamma \mu \rho)$ then $a_{2}>0$. Since $\frac{c \beta \lambda}{\alpha \gamma \delta}=\frac{\mu}{\delta} R_{0}$, 


\section{APPLICATION TO THE MODEL}

if $R_{0}>\frac{\delta}{\mu}$ then $c \beta \lambda>\alpha \gamma \delta$. Thus, if $c \beta>\alpha \rho$ and $R_{0}>\frac{\delta}{\mu}$ then $c \beta \lambda>\alpha \gamma \delta$. Therefore, if $R_{0}>\frac{\delta}{\mu}$ and $\lambda(\gamma+\delta)>\alpha \delta^{2}$ then $a_{2}>0$.

Considering the numerator of $a_{3}$, we want to prove that $\delta\left(-\alpha \gamma^{2} \mu \rho^{2}(\gamma+\mu)+\beta^{2} c^{2}\left(\lambda \rho(\gamma+\delta)-\alpha \delta^{2}\right)+\beta c \gamma \rho(\lambda \rho(\gamma+\right.$ $\mu)-\alpha \delta(\gamma+2 \mu)))>0$. Additionally, if $\beta c \delta \lambda \rho(\beta c(\gamma+\delta)+\gamma \rho(\gamma+\mu))>\alpha \delta\left(\beta^{2} c^{2} \delta^{2}+\gamma^{2} \mu \rho^{2}(\gamma+\mu)+\beta c \gamma \delta \rho(\gamma+2 \mu)\right)$, then $a_{3}>0$. Thus, if $R_{1}>1$, and $\lambda \rho(\gamma+\delta)>\alpha \delta^{2}$, then $a_{3}>0$.

Furthermore, $a_{4}$ can be written as

$$
a_{4}=\frac{\delta}{\rho(c \alpha \beta \delta+\alpha \gamma \mu \rho)}\left(R_{1}-1\right)
$$

Thus, if $R_{1}>1$ then $a_{4}>0$; however, if $R_{1}<1$, then $a_{4}<0$ and $P_{3}$ is unstable.

The expression for $a_{1} a_{2} a_{3}-\left(a_{3}^{2}+a_{1}^{2} a_{4}\right)$ can be found in the appendix. However, if $c \beta>\alpha \rho, c \beta \lambda>\alpha \delta \gamma, R_{1}>$ $1, R_{0}>1$, and $\lambda \rho(\gamma+\delta)>\delta^{2} \alpha$, then $a_{1} a_{2} a_{3}-\left(a_{3}^{2}+a_{1}^{2} a_{4}\right)>0$ and thus, $a_{1} a_{2} a_{3}>\left(a_{3}^{2}+a_{1}^{2} a_{4}\right)$.

Figure 8 illustrates the dynamic between the virus and uninfected cell populations given that $R_{0}, R_{1}>1$. Based on our analysis, we expect a stable viral persistent equilibrium that maintains an immune response. The graph shows the persistence of the virus as $t \rightarrow \infty$ as the viral load remains positive. Furthermore, Figure 8 suggests that the system approaches a viral and immune response persistence equilibrium, highlighting the local asymptotic stability predicted by our analysis. This behavior is consistent with our analysis given $R_{0}, R_{1}>1$.

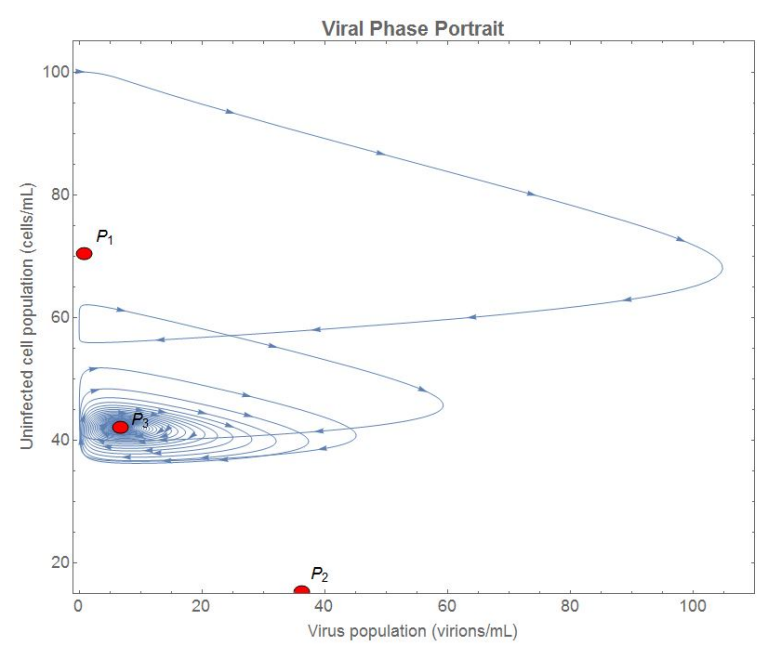

Figure 8: A system phase portrait for $R_{0}=4.6864$ and $R_{1}=2.8119$.
Similar to Figure 7, the virus population appears to approach zero several times during the course of infection, reaching very low levels within the system. This may suggest the presence of a latency period. However, in Figure 9 there appears to be significantly more oscillation between populations than in Figure 7. This may suggest that as the magnitude of $R_{0}$ and $R_{1}$ increase, the dynamical behavior will increase as well. However, there is a possibility that the behavior may be solely a result of the magnitude of $R_{1}$ since in Figure $6, R_{1}<1$ whereas in Figure $9, R_{1}>1$. Thus, $R_{1}$ may have an effect on the oscillatory behavior of the system. The behavior in Figure 8 suggests that the virus is able to reinfect the host multiple times, even once the population approaches zero during infection. Thus, the magnitude of $R_{0}$ and $R_{1}$ may have an effect on the reactivation and re-emergence of the virus from a latency period.

Figure 9 displays the relationship between the virus and the CTL response. Again, the figure illustrates that the equilbirum is stable, as we expected. Additionally, as $t \rightarrow \infty$ the immune response remains non-zero suggesting that the immune response is present even when the populations stabilize. In addition, this figure depicts the oscillations described above. Furthermore, Figure 9 illustrates

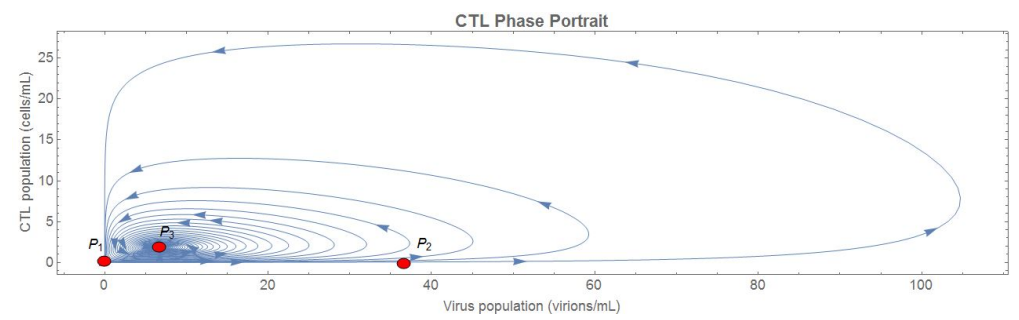

Figure 9: A CTL phase portrait for $R_{0}=4.6864$ and $R_{1}=2.8119$. 


\section{APPLICATION TO THE MODEL}

that the immune response occurs only when the virus is close to the peak viral load point, which is what we expect given the known dynamics of viral infection. However, Figure 10 reveals that the magnitude of the immune response remains relatively small when compared with the population of uninfected cells in Figure 9. This again may suggest that the virus is evading a full response from the immune system.

Figure 10 displays the dynamic between the virus, uninfected cell population, and the CTL response. The figure illustrates that the equilibrium is stable, as we expected given that both $R_{0}$ and $R_{1}>1$. Additionally, the equilibrium maintains both a non-zero virus population as well as an immune response. However, the immune response, as reflected by the CTL population, appears to be limited and insubstantial.

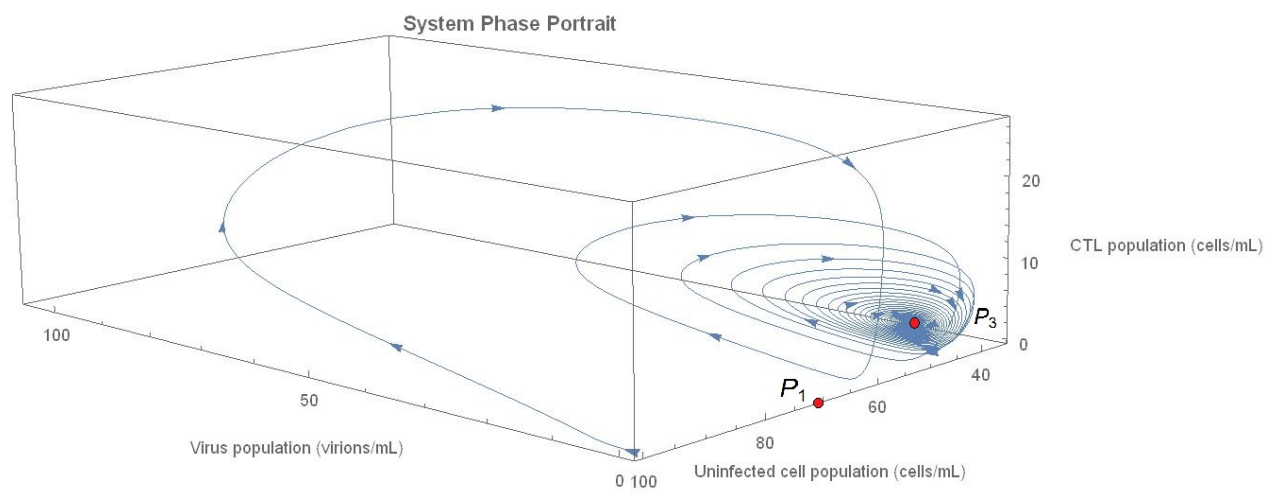

Figure 10: A system phase portrait for $R_{0}=4.6864$ and $R_{1}=2.8119$.

\subsection{Model Simulations}

Upon examination it is clear that the values of the viral reproduction numbers, $R_{0}$ and $R_{1}$, have large effects on the behavior of the overall system. Our analysis demonstrates that a comparison of these values determined which equilbiria were biologically feasible as well as the local asymptotic stability behavior at these values. By understanding the stabilities of the solutions, we were able to determine viral persistence or elimination as well as the persistence or extinction of other cellular populations. However, the simulation reveals that the magnitude of both values also greatly changes not only the long term behavior, but also has a large impact on the dynamics of the system during the course of infection. In addition, the simulations reveal and illustrate some interesting insights into the behavior of the virus which have been recently determined. More specifically, the simulations reveal the ability of Ebola virus to evade detection by the immune system. In Figure 11 graph (a), $R_{0}$ and $R_{1}$ are both greater than one. Thus, we expect to obtain a stable equilibrium which is viral-persistent. In addition, we expect that the equilibrium also maintains an immune response as $t \rightarrow \infty$. In Figure 11 graph (b), the value for $R_{0}$ is greater than one, however, the value of $R_{1}$ is less than one. Thus, we expect to obtain a stable viral persistence equilibrium. For both Figure 11 graphs $(c)$ and $(d)$ the values of $R_{0}$ and $R_{1}$ are less than one and thus we expect that the equilbirium will be a virus free equilibrium and that the only population that remains as $t \rightarrow \infty$ is the population of uninfected cells.

Figure 11 shows the system dynamic interaction plots for four different combinations of $R_{0}$ and $R_{1}$ defined in the table below. These plots illustrate how all of the populations are interacting with one another over time. 


\section{APPLICATION TO THE MODEL}
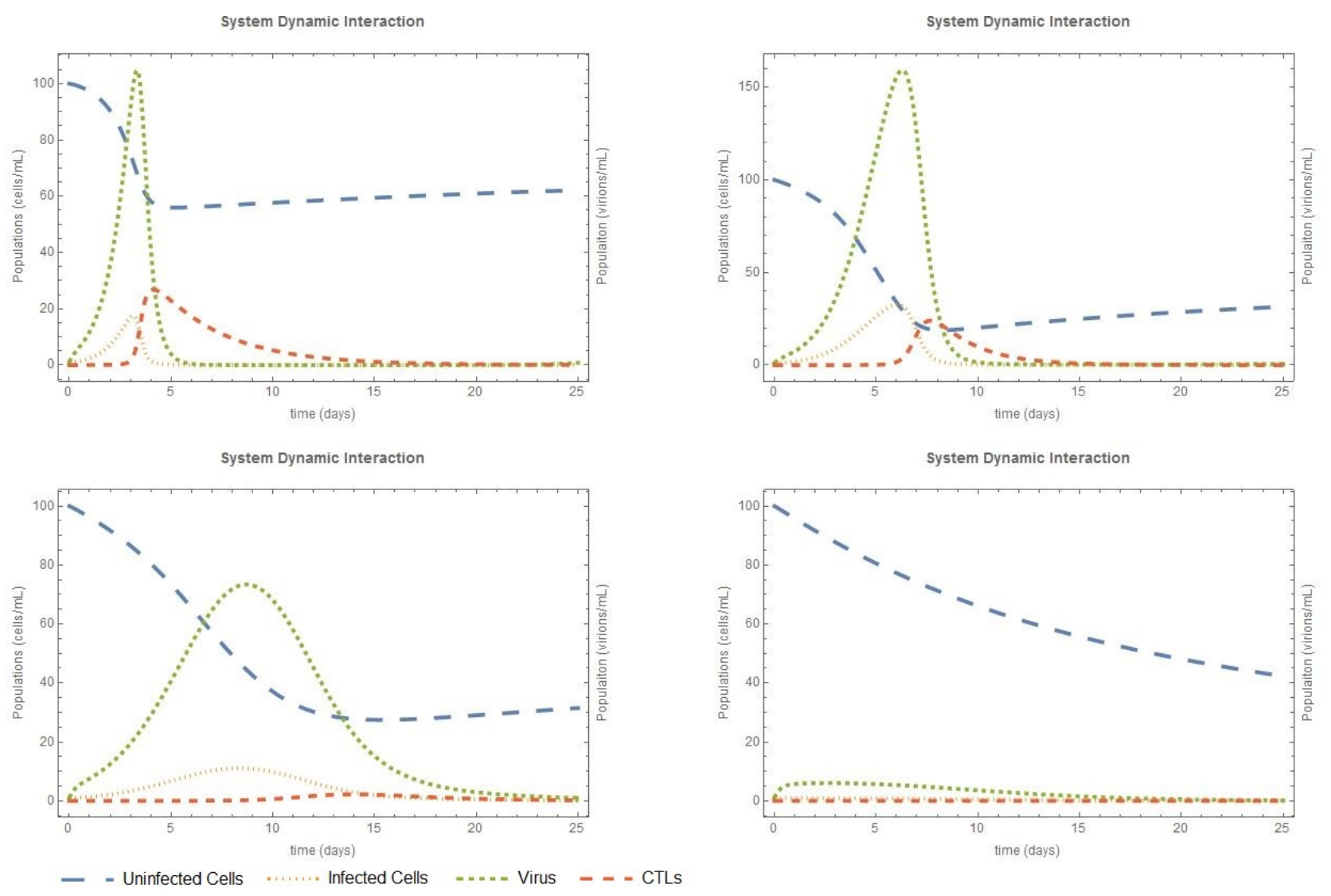

Figure 11: A comparison of the course of viral infection and immune response based on differing values of $R_{0}$ and $R_{1}$.

\begin{tabular}{|c|c|c|c|}
\hline Location & Value of $R_{0}$ & Value of $R_{1}$ & Corresponding Phase Portraits \\
\hline upper-left (a) & 4.6864 & 2.8119 & Figures $8,9,10$ \\
\hline upper-right (b) & 2.1089 & 0.8436 & Figures $5,6,7$ \\
\hline lower-left (c) & 0.9373 & 0.5207 & Figures 2, 3 \\
\hline lower-right (d) & 0.2464 & 0.1760 & Figure 4 \\
\hline
\end{tabular}

The parameters for the plots in Figure 11 were chosen from the ranges provided in the parameter table in section two to achieve specified values of $R_{0}$ and $R_{1}$. We then simulated the system in Mathematica and provide the obtained plots from our simulations. In Figure 11 graphs (a), (b), and (c), we can clearly see the proliferation of the virus upon introduction at time zero. In all three cases, the virus reaches a peak viral load point within the first few days of infection; this behavior is characteristic of Ebola infection [1]. For instance Figure 11 graph (a) reaches a peak viral load of $104.735 \frac{\text { virions }}{m L}$ at day 3.324 and graph (b) achieves its peak of $159.22 \frac{\text { virions }}{m L}$ at day 6.280. Yet, we can clearly see that the time in which the peak viral load point is achieved, as well as the magnitude of the viral load, is influenced by the values of $R_{0}$ and $R_{1}$. It appears that when $R_{0}$ and $R_{1}$ increase, the infection progresses at a much faster rate. However, we can see that in Figure 11 graph (b) the peak viral load point is $54.485 \frac{\text { virions }}{m L}$ greater than that of Figure 11 graph (a). This may be explained by the value of $R_{1}$. As we mentioned previously, $R_{1}$ serves to translate the notion of viral reproductive numbers to immune response. In this manner, $R_{1}$ represents the number of infected cells that a single immune cell (CTL in the case of our model) is able to address. Thus, a greater $R_{1}$ value would be associated with a smaller peak in the infected cell population, and thus a smaller peak viral load point which is precisely the behavior we see 


\section{DISCUSSION AND CONCLUSIONS}

illustrated by Figure 11 graphs (a) and (b).

In addition, we see the massive effect that infection has on the population of the uninfected cells. In Figure 11 graphs (b) and (c) the population of uninfected cells is reduced to $31.456 \%$ and $31.497 \%$ of its original size by day 25 of infection respectively. Furthermore, for graph (a) the population is reduced to $62.04 \%$ of its original size by day 25 . Yet, in each case the uninfected cell population remains strictly positive, as expected. It is important to note that during infection the population is never completely eliminated.

Figure 11 graphs (a) and (b) illustrate viral persistence, however, the population of the virus is extremely low within the system, approximately $0.005 \frac{\text { virions }}{m L}$. As mentioned previously, this may suggest the potential for a latency period in which the virus remains dormant within the immune system. Figure 11 graphs (c) and (d) highlight the notion of viral extinction. In these two cases, the virus is eliminated from the system as $t \rightarrow \infty$.

Figure 11 graph (d) illustrates the case in which Ebola virus is never able to reach a critical infection point; thus, the virus is not able to sustain the infection. In other words, the virus is not "infectious" enough in order to establish itself within the system. From a biological perspective, this means that each virus particle is not able to infect a cell and produce a new virus particle, and thus the infection is eliminated in the long run. There are several possible methods by which this can occur. First, the virus is able to be eliminated via regular cell death. Thus, there is no development of an immune response during the infection. However, the population of the virus may also remain at a low enough level that the immune system either does not recognize the infection prior to elimination, or the required response is minimal and virtually undetectable.

The most important result from the simulations is the notion of immune response. In each case, the immune system does not seem to mount a prolific, or appropriate, response to the infection. In an ideal scenario, the immune system would recognize the pathogen immediately after infection and begin a response by dividing the specific T-cell or B-cell which recognized the pathogen, ultimately resulting in a dramatic increase in the population of CTLs. However, the population of CTLs remains relatively low during the course of infection when compared to the other cellular populations, or the CTLs are altogether non-existent within the system. This suggests that regardless of the values of $R_{0}$ and $R_{1}$, the virus is able to evade detection and response, which is notably the exact behavior of the Ebola virus during infection.

For instance, in Figure 11 graphs (a) the immune response is $26.753 \frac{\text { cells }}{m L}$ at its peak point (day 4.13) and $23.994 \frac{\mathrm{cells}}{\mathrm{mL}}$ (day 7.67) in graph (b). This magnitude of response is significantly lower than the population of uninfected cells, and is much smaller than immune responses in other typical viral infections, including the early stages of human immunodeficiency virus (HIV) (prior to the onset of acquired immune deficiency syndrome, or AIDS) and chicken-pox infections. In graph (c), the immune response is barely present and only occurs late in the infection, and in Figure 11 graph (d) there is no immune response development highlighted by the absence of CTLs. Furthermore, in all cases the duration of the immune response is extremely short-lived.

\section{Discussion and Conclusions}

In this paper we develop and analyze a model for Ebola virus dynamics in vivo using reasonable biological assumptions about both the virus and its primary cellular target in mammalian cells. The proposed model focused on the highly dynamic interaction between Ebola virus particles, uninfected cells typically prone to infection by Ebola, infected cells and CTLs. The development and analysis of a model which incorporates the immune response to viral infection was a key feature in examining the course of Ebola infection within the human immune system.

Our findings illustrate that utilizing two determined threshold parameters, $R_{0}$ known as the viral reproduction number, and $R_{1}$ determined to be the immune response number, as well as some small, biologically reasonable assumptions, allow us to predict the local asymptotic stability of the system around the equilibria. Furthermore, we are able to classify equilibria based upon their persistence or extinction of specific cell types or virions. This is especially important when considering the notion of viral persistence and extinction as we are able to determine conditions which yield viral extinction and persistence as well as immune extinction and persistence. Our analysis highlights that if the value of the viral reproduction number, $R_{0}$, is less than one, the equilibrium will be a locally asymptotically stable virus-free equilibrium. This insight is consistent for that of other viral infections, including the human immunodeficiency virus (HIV) $[15,16,20,21,26]$. In addition, 


\section{DISCUSSION AND CONCLUSIONS}

our analysis illustrated that increasing the value of $R_{1}$ such that $R_{1}>1$, is consistent with a decrease in the peak viral load as well as a decrease in the peak population of virally infected cells. Thus, using the theorems we developed, we are able to establish conditions which by incorporating the values of $R_{0}$ and $R_{1}$ are able to predict the long term outcomes of Ebola virus infection.

Furthermore, our findings illustrate the notion of evasion of the virus from the immune system. Our simulations revealed that the population of the CTLs remains extremely low when compared to the other cellular populations. This type of behavior is typically exhibited by other viral infections which subvert immune responses. In the case where $R_{0}<1$ this behavior is expected from most viral infections, and results from the fact that each infected cell will fail to produce an additional infected cell prior to death. In this sense, the virus is not "infectious" enough to commence and sustain an infection which will necessitate a robust immune response. However, when $R_{0}>1$ we fully expect that the immune system will become activated and thus, we would expect an increase in the CTL population [26]. We believe that this behavior exhibited by the model comes as a result of the ability of Ebola virus to effectively evade immune detection.

It is important to illustrate that our biological understanding of Ebola virus infection agrees with the mathematics we have shown above. We know from the underlying biology that Ebola virus maintains the ability to evade both host innate and humoral responses. As mentioned previously, each Ebola virus particle consists of the polymerase cofactor VP35, the virus specific transcription activator VP30, and the viral RNA polymerase L proteins [3]. In addition, the matrix between the outer viral envelope and the nucleocapsid contains the VP40 and VP24 viral proteins [3]. It is via these proteins that Ebola virus is able to subvert the immune response. Ebola virus evades host innate immune responses by downregulation of type I inferion (IFN) responses with VP24, which desensitize host cells to the effects of IFN- $\alpha / \beta$ and IFN- $\gamma$, and VP35, which can interfere with the synthesis and expression of IFN and inferion stimulated genes. Furthermore, the Ebola virus glycoprotein (GP) uses epitope masking and seric shielding to prevent interactions of host MHC Class I and $\beta 1$ integrin thereby inhibiting the host immune response. In addition, Ebola evades host humoral immune responses through antigenetic subversion with soluble GP and prevention of dendritic cell maturation with VP35. While modeling these detailed and complex interactions would be extremely difficult, the general behavior of the virus and the immune system resulting from our model is highly consistent with what we know about dynamics of the infection.

Furthermore, our work in this paper reveals that the model we developed effectively addresses the dynamics of Ebola virus infection in vivo, including an immune response. Thus, there is potential to further the analysis that we conducted in this paper to incorporate a time delay, which would more accurately model the true dynamics of the system. Lastly, we could also introduce various treatment regiments or protocols and analyze their impact on the behavior of the system.

\section{Appendix}

To prove the existence and uniqueness of solutions, we must prove the following theorem, referred to in Section 3.1.

Theorem (Fundamental Existence and Uniqueness Theorem). Suppose the function $f: \mathbb{R}^{n} \rightarrow \mathbb{R}^{n}$ is continuously differentiable. Then $x(t)$ is a solution of the differential equation $\frac{d x}{d t}=f(x)$ on an interval I if $x(t)$ is differentiable on $I$ and if $\forall t \in I, x(t) \in \mathbb{R}^{n}$ and $\frac{d x}{d t}=f(x(t))$ and given $x_{0} \in \mathbb{R}^{n}, x(t)$ is a solution of the initial value problem

$$
\begin{aligned}
& \frac{d x}{d t}=f(x) \\
& x\left(t_{0}\right)=x_{0}
\end{aligned}
$$

Remark: The above is a well known theorem and ensures that the solutions exists and is unique in the neighborhood of $x_{0}$, i.e., the function is locally Lipschitz [21]. The proof for this theorem can be found in [17].

For determining the local stability behavior of the system at the equilibria of a non-linear system, the following theorem is necessary: 


\section{DISCUSSION AND CONCLUSIONS}

Theorem (Poincare-Perron). Let $A$ be a constant matrix in the system $\frac{d x}{d t}=A x$ with eigenvalues $\lambda_{i}, i=$ $1,2, \ldots, n$

i. If the system is stable, then $\operatorname{Re}\left\{\lambda_{i}\right\} \leq 0 . i=1,2, \ldots, n$.

ii. If either $\operatorname{Re}\left\{\lambda_{i}\right\}<0, i=1,2, \ldots, n$; or if $\operatorname{Re}\left\{\lambda_{i}\right\} \leq 0 i=1,2, \ldots, n$ and there is no zero repeated eigenvalue; then the system is uniformly stable.

iii. The system is asymptotically stable if and only if $\operatorname{Re}\left\{\lambda_{i}\right\}<0, i=1,2, \ldots, n$; note that it is also uniformly stable by ii.

iv. If $\operatorname{Re}\left\{\lambda_{i}\right\}>0$, for any $i=1,2, \ldots, n$ the solution is unstable.

Remark: If any of the eigenvalues have a positive real numbers, we define the equilibrium to be a source, and thus, unstable. If all of the real parts of the eigenvalues are negative real numbers, we define the equilibrium to be a sink, and thus, stable. The proof for this theorem can be found in [17].

To determine the signs of the eigenvalues without solving the system directly, we use the Routh-Hurwitz stability criterion.

Theorem (Routh-Hurwitz Criteria). Given the polynomial $P(x)=x^{n}+a_{1} x^{n-1}+\ldots+a_{n-1} x+a_{n}$, where the coefficients $a_{i}$ are real constants, $i=1, \ldots, n$, define the $n^{\text {th }}$ Hurwitz matrix using the coefficients $a_{i}$ of the characteristic polynomial:

$$
H_{n}=\left[\begin{array}{cccccc}
a_{1} & 1 & 0 & 0 & \cdots & 0 \\
a_{3} & a_{2} & a_{1} & 1 & \cdots & 0 \\
a_{5} & a_{4} & a_{3} & a_{2} & \cdots & 0 \\
\vdots & \vdots & \vdots & \vdots & \cdots & \vdots \\
0 & 0 & 0 & 0 & \cdots & a_{n}
\end{array}\right]
$$

where $a_{i}=0$ if $j>n$. All of the roots of the polynomial $P(x)$ are negative or have negative real part iff the determinants of all Hurwitz matricies are positive: $\operatorname{det}\left(H_{j}\right)>0, j=1,2, \ldots, n$.

Considering $n=3$ and $n=4$, the theorem simplifies and we are able to apply the theorem to the analysis of our system. For $n=3$ the following conditions must be met: $a_{1}>0, a_{3}>0, a_{1} a_{2}>a_{3}$. For $n=4$ we must prove that: $a_{1}>0, a_{3}>0, a_{4}>0, a_{1} a_{2} a_{3}>a_{3}^{2}+a_{1}^{2} a_{4}$.

The Hartman-Grobman theorem is essential for showing how our analysis of the linearized system relates to the non-linear system.

Theorem (The Hartman-Grobman Theorem). Let $E$ be an open subset of $\mathbb{R}^{n}$ containing the origin, let $f \in$ $C^{2}(E)$ and let $\phi_{t}$ be the flow of the nonlinear system below. Suppose that $f(0)=0$ and that the Jacobian matrix $J(0)$ has no eigenvalue with zero real part. Then there exists a homeomorphism $H$ of an open set $U$ containing the origin onto an open set $V$ containing the origin such that for each $x_{0} \in U$ there is an open interval $I_{0} \subset \mathbb{R}$ containing zero such that for all $x_{0} \in U$ and $t \in I_{0}$

$$
H \circ \phi_{t}\left(x_{0}\right)=e^{A t} H\left(x_{0}\right)
$$

Note: The proof for this theorem can be found in [17].

In our analysis for $P_{3}$ from section 4.4.1 we also need to show that $a_{1} a_{2} a_{3}-\left(a_{3}^{2}+a_{1}^{2} a_{4}\right)>0$. We know for $P_{3}$ : $a_{1} a_{2} a_{3}-\left(a_{3}^{2}+a_{1}^{2} a_{4}\right)=\frac{1}{\gamma^{3} \rho^{2}(\beta c \delta+\gamma \mu \rho)^{3}}\left(\beta c \delta \lambda\left(\alpha \gamma^{5} \mu^{2} \rho^{5}(\gamma+\mu)\left(\alpha \delta-\mu^{2}\right)+\beta^{5} c^{5} \delta^{3}\left(\delta(\lambda \rho-\alpha \delta)+\gamma^{2} \delta+\gamma \lambda \rho\right)+\right.\right.$

$\beta^{4} c^{4} \gamma \delta \rho\left(\delta(\lambda \rho(4 \delta \mu+\lambda \rho)-\alpha \delta(5 \delta \mu+\lambda \rho))+\gamma^{3} \delta^{2}+\gamma^{2} \delta(4 \delta \mu+\lambda \rho)+\gamma \lambda \rho(3 \delta \mu+\lambda \rho)\right)+\beta^{3} c^{3} \gamma^{2} \rho^{2}\left(\alpha^{2} \delta^{4}+\right.$ $\alpha \delta^{2}\left(\gamma^{2} \delta-\gamma(\delta \mu+\lambda \rho)-2 \delta\left(\lambda \rho+5 \mu^{2}\right)-3 \lambda \mu \rho\right)+3 \gamma^{3} \delta^{2} \mu+\gamma^{2} \delta\left(-\delta \lambda \rho+6 \delta \mu^{2}+3 \lambda \mu \rho\right)+\gamma \lambda \rho\left(\delta^{2} \mu+\delta \lambda \rho+3 \delta \mu^{2}+\right.$ $\left.\lambda \mu \rho)+\delta \lambda \rho\left(\delta \lambda \rho+6 \delta \mu^{2}+2 \lambda \mu \rho\right)\right)+\beta^{2} c^{2} \gamma^{3} \rho^{3}\left(\alpha^{2} \delta^{3}(\gamma+3 \mu)+\alpha \delta\left(2 \gamma^{2} \delta \mu-\gamma\left(2 \delta \lambda \rho+3 \delta \mu^{2}+\lambda \mu \rho\right)-\mu(4 \delta \lambda \rho+\right.\right.$ $\left.\left.\left.10 \delta \mu^{2}+3 \lambda \mu \rho\right)\right)+3 \gamma^{3} \delta \mu^{2}+\gamma^{2} \mu\left(-\delta \lambda \rho+4 \delta \mu^{2}+2 \lambda \mu \rho\right)+\gamma \lambda \rho\left(\delta \lambda \rho+2 \delta \mu^{2}+\mu^{3}\right)+\lambda \mu \rho\left(\delta \lambda \rho+4 \delta \mu^{2}+\lambda \mu \rho\right)\right)+$

$$
\left.\beta c \gamma^{4} \mu \rho^{4}\left(\alpha^{2} \delta^{2}(2 \gamma+3 \mu)+\alpha\left(\gamma^{2} \delta \mu-\gamma \delta\left(2 \lambda \rho+3 \mu^{2}\right)-\mu\left(2 \delta \lambda \rho+5 \delta \mu^{2}+\lambda \mu \rho\right)\right)+\mu^{2}(\gamma+\mu)\left(\gamma^{2}+\lambda \rho\right)\right)\right)
$$

thus, since $c \beta>\alpha \rho, c \beta \lambda>\alpha \delta \gamma, R_{1}>1, R_{0}>1$, and $\lambda \rho(\gamma+\delta)>\delta^{2} \alpha$ we can simplify the expression to show that $a_{1} a_{2} a_{3}>\left(a_{3}^{2}+a_{1}^{2} a_{4}\right)$ and thus we have shown $a_{1} a_{2} a_{3}-\left(a_{3}^{2}+a_{1}^{2} a_{4}\right)>0$. 


\section{REFERENCES}

\section{References}

[1] Audet, J., and G. P. Kobinger. "Immune Evasion in Ebolavirus Infection." Viral Immunology 28, no. 1 (February 28, 2015): 10-18.

[2] Banton, Sophia, Zvi Roth, and Mirjana Pavlovic. "Mathematical Model of Ebola Virus Dynamics as a Step towards Rational Vaccine Design." IFMBE 32 (2010).

[3] Baylor College of Medicine. "Ebola Virus." Molecular Virology and Microbiology. Accessed November 29, 2014. https://www.bcm.edu/departments/molecular-virology-and-microbiology/emerging-infections-andbiodefense/ebola-virus.

[4] Carter, John, and Venetia Saunders. Virology: Principles and Applicaitons. 2nd ed. N.p.: Wiley and Sons, 2013.

[5] Centers for Disease Control and Prevention. "2014 Ebola Outbreak in West Africa - Case Counts." Ebola (Ebola Virus Disease). Last modified March 22, 2015. Accessed March $13,2015$. http://www.cdc.gov/vhf/ebola/outbreaks/2014-west-africa/case-counts.html.

[6] Chowell, G., N. Hengartner, C. Castillo-Chavez, P. Fenimore, and J. Hyman. "The basic reproductive number of Ebola and the effects of public health measures: the cases of Congo and Uganda." Journal of Theoretical Biology 229, no. 1 (July 7, 2004): 119-26.

[7] Culshaw, Rebecca V., and Shigui Ruan. "A delay-differential equation model of HIV infection of CD4+ T-cells." Mathematical Biosciences 165 (February 2000): 27-39.

[8] Hubbard, J. H., and B. H. West. Differential Equations: A Dynamical Systems Approach. Texts in Applied Mathematics 18. New York, NY: Springer-Verlag, 1995.

[9] Jordan, D. W., and P. Smith. Nonlinear Ordinary Differential Equations. 4th ed. New York, NY: Oxford University Press, 2007.

[10] Lakshmanan, M., and D. V. Senthilkumar. Dynamics of Nonlinear Time-Delay Systems. Berlin, Germany: Springer, 2010 .

[11] Lentz, and Feezor. "Principles of Immunology." Nutrition in clinical practice: official publication of the American Society for Parenteral and Enteral Nutrition 18, no. 6 (December 2003).

[12] Mohan, Gopi S., Wenfang Li Ling Ye, Richard W. Compans, and Chinglai Yang. "Antigenic Subversion: A Novel Mechanism of Host Immune Evasion by Ebola Virus." PLOS Pathogens 10 (December 13, 2012).

[13] Murray, John D. An Introduction. 3rd ed. Vol. I of Mathematical Biology. Berlin: Springer, 2003. http://www.cancer.gov/cancertopics/factsheet/Risk/hiv-infection.

[14] Owen, Judy, Jenni Punt, and Sharon Stranford. Kuby Immunology. 7th ed. N.p.: W.H. Freeman, 2013.

[15] Perelson, Alan. "Modelling Viral and Immune System Dynamics." Nature Reviews, January 2002, 28-36.

[16] Perelson, Alan, and Patrick Nelson. "Mathematical Analysis of HIV-1 Dynamics in Vivo." Society for Industrial and Applied Mathematics 41, no. 1 (March 2001): 3-43.

[17] Perko, Lawrence. Differential Equations and Dynamical Systems. Texts in Applied Mathematics 7. New York, NY: Springer, 2006.

[18] Peters, C. J., and J. W. LeDuc. "An Introduction to Ebola: The Virus and the Disease." Journal of Infectious Diseases, no. 179 (1999).

[19] Reece, Jane, Lisa Urry, Michael L. Cain, Steven A. Wasserman, Peter V. Minorsky, and Robert B. Jackson. Campbell Biology. 9th ed. New York: Benjamin Cummings, 2010.

[20] Rihan, Fathalla A., and Duaa H. Abdel Rahman. "Delay differential model for tumor-immune dynamics with HIV infection of CD4+ T-cells." International Journal of Computer Mathematics 90, no. 3 (2013): 594-614.

[21] Roemer, Peter A. "Stochastic Modeling of the Persistence of HIV: Early Population Dynamics." Trident Scholar Project Report. Last modified 2013.

[22] Srivastava, Prashant, and Peeyush Chandra. "Modeling the dynamics of HIV and CD4+ T cells during primary infection." Nonlinear Analysis: Real World Applications 10 (October 8, 2008).

[23] Sullivan, Nancy, Zhi-Yong Yang, and Gary J. Nabel. "EbolaVirus Pathogenesis: Implications for Vaccines and Therapies." Journal of Virology 77, no. 18 (September 2003).

[24] Truckwell, Henry, and Frederic Wan. "Nature of equilibria and effects of drug treatments in some simple viral population dynamical models. IMA Journal of Mathematics Applied in Medicine and Biology 17 (June 2000): 31127.

[25] University of Vermont College of Engineering and Mathematical Sciences. "Routh-Hurwitz Criteria." Math 295: Systems Theory. Accessed December 6, 2014. http://www.cems.uvm.edu/t̃lakoba/08fall/EE295/.

[26] Wester, Thomas, Daniel Isaac, and Sonia Garcia. "Mathematical Modeling: Immune System Dynamics in the Presence of Cancer and Immunodeficiency in vivo." Working paper, Department of Mathematics, United States Naval Academy, Annapolis, MD, 2015. 